Journées Francophones de Nutrition (Montpellier, 29 Novembre-2 Décembre 2016)

\title{
Découverte de métabolites prédictifs du risque de cancer du sein : approche métabolomique RMN appliquée à l'épidémiologie nutritionnelle
}

\author{
Lécuyer L, Victor Bala A, Deschasaux M, Bouchemal N, Triba M, Hercberg S, Galan P, \\ Kesse-Guyot E, Latino-Martel P, Fezeu LK, Savarin P, Touvier M.
}

\section{Résumé :}

Introduction et but de l'étude : La métabolomique est une science émergeante qui étudie l'ensemble des métabolites présents dans une cellule, un organe, un biofluide. Son application au domaine de l'épidémiologie nutritionnelle ouvre des perspectives considérables. A notre connaissance, aucune étude prospective n'avait été menée pour investiguer les liens entre les profils métabolomiques non-ciblés à l'inclusion et le risque de développer un cancer du sein à long terme. Ce projet propose donc pour la 1ère fois d'étudier si des signatures métabolomiques établies à partir d'un simple prélèvement sanguin pourraient contribuer à mieux comprendre et à prédire le risque de cancer du sein dans la décennie suivante.

Matériel et méthodes : Une étude cas-témoin nichée a été mise en place dans la cohorte SU.VI.MAX (1994-2007), incluant 206 cas de cancer du sein et 396 témoins appariés. Les profils métabolomiques RMN ont été établis sur des échantillons de plasma prélevés à l'inclusion (donc avant l'apparition des cancers). Des régressions logistiques conditionnelles multivariées ont été utilisées. La performance prédictive des modèles a été évaluée grâce au NRI (Net Reclassification Improvement).

Résultats et Analyse statistique : 237 buckets ont été obtenus après division du spectre RMN par « intelligent bucketing », dont 25 étaient significatifs dans les modèles logistiques pour la séquence NOESY (respectivement 228 buckets et 27 significatifs pour la séquence CPMG). Les probabilités critiques correspondantes allaient de 0,00007 (pour le bucket 5,1869ppm, correspondant au groupement méthine du glycéryl, ORT3vs.T1=0,37 [0,23-0,61]) à 0,04 (pour le bucket 2,429ppm, correspondant à la glutamine, ORT3vs.T1=1,62 [1,02-2,57]). Des lipoprotéines, des lipides (dont des acides gras insaturés et des glycérides et/ou des phosphoglycérides et dérivés) et des glycoprotéines étaient associés à une diminution du risque de développer un cancer du sein alors que plusieurs acides aminés et dérivés (Valine, Leucine, Glutamine, Créatine, Créatinine et la Thréonine) et le bêta-glucose étaient associés à une augmentation du risque. La plupart de ces métabolites augmentaient significativement la performance prédictive des modèles.

Conclusion : Cette étude pionnière suggère que plusieurs métabolites, dont certains appartenant au food metabolome, seraient impliqués dans l'étiologie du cancer du sein. Des analyses similaires en métabolomique par spectrométrie de masse sont en cours dans l'étude, ainsi que l'étude des corrélations entre les profils métabolomiques et les apports nutritionnels. 